Cell Research (2003); 13(2):111-120

http://www.cell-research.com

\title{
Interaction with general transcription factor IIF (TFIIF) is required for the suppression of activated transcription by RPB5-mediating protein (RMP)
}

Wenxiang WEI ${ }^{1, *}$, Jun Xia GU² Cui Qing ZHU ${ }^{1}$, Feng Yan SUN ${ }^{1}$ ，DorJbal DORJSUREN ${ }^{3}$, Yong LIN $^{3}$, SEISHI MURAKAMI ${ }^{3, * *}$

${ }^{1}$ National Key Laboratory of Medical Neurobiology, Fudan University, 138 Yi Xue Yuan Road, Shanghai 200032, China

${ }^{2}$ Department of Hematology, the Affiliated Xiehe Hospital, Tongji Medical School, Huazhong University ofScience and Technology, 1277 Jiefang Avenue, Wuhan 430022, China

${ }^{3}$ Department of Molecular Oncology, Cancer Research Institute, Kanazawa University, Takara-machi 13-1, Kanazawa 920-0934, Japan

\begin{abstract}
RMP was reported to regulate transcription via competing with $\mathrm{HBx}$ to bind the general transcription factor IIB (TFIIB) and interacting with RPB5 subunit of RNA polymerase II as a corepressor of transcription regulator. However, our present research uncovered that RMP also regulates the transcription through interaction with the general transcription factors IIF (TFIIF), which assemble in the preinitiation complex and function in both transcription initiation and elongation. With in vitro pull-down assay and Far-Western analysis, we demonstrated that RMP could bind with bacterially expressed recombinant RAP30 and RAP74 of TFIIF subunits. In the immunoprecipitation assay in COS1 cells cotransfected with FLAG-tagged RMP or its mutants, GST-fused RAP30 and RAP74 were co-immunoprecipitated with RMP in approximately equal molar ratio, which suggests that RAP30 and RAP74 interact with RMP as a TFIIF complex. Interestingly both RAP30 and RAP74 interact with the same domain (D5) of the C-terminal RMP of 118-aminoacid residuals which overlaps with its TFIIB-binding domain. Internal deletion of D5 region of RMP abolished its binding ability with both subunits of TFIIF, while D5 domain alone was sufficient to interact with TFIIF subunits. The result of luciferase assay showed that overexpression of RMP, but not the mutant RMP lacking D5 region, suppressed the transcription activated by Gal-VP16, suggesting that interaction with TFIIF is required for RMP to suppress the activated transcription. The interaction between RMP and TFIIF may be an additional passway for RMP to regulate the transcription, or alternatively TFIIF may cooperate with RPB5 and TFIIB for the corepressor function of RMP.
\end{abstract}

Key words: general transcription factor IIF, RPB5-mediating protein, activated transcription, RNA polymerase II subunit 5 .

*Present address: Department of Biochemistry, University of Medicine and Dentistry of New Jersey, Robert Wood Johnson Medical School, Piscataway, NJ 08854, USA. Tel: 001-(732)235-4194.E-mail:weiwe@umdnj.com

**Correspondence:Dr. Seishi MURAKAMI

Tel: 0081-76-265-2731 Fax: 0081-76-234-4501

E-mail: semuraka@kenroku.ipc.kanazawa-u.ac.jp

Abbreviations: pol I, II, and III, RNA polymerase I, II, and III, respectively; TF, transcription factor; $\mathrm{HBx}$, hepatitis B virus $\mathrm{X}$ protein; RPB, RNA polymerase II subunit; GST, glutathione S-transferase; PAGE, polyacrylamide gel electrophoresis; RMP, RPB5-mediating protein; PCR, polymerase chain reaction. Received Dec-27-2002 Revised Jan-18-2003 Accepted Feb-24-2003

\section{INTRODUCTION}

RNA polymerase II (pol II) transcribes mRNA and small nuclear RNA. The subunits of RNA polymerase II have been the final targets of variety of factors to regulators gene transcription[1-4]. Pol II consists of 12 subunits of which RPB5 is the common subunit of pol I, II and III[4]. Evidence accumulated that RPB5 is one of active subunit of pol II 
during the gene transcription. $\mathrm{HBx}$ was found to target RPB5 to stimulate transactivation[5]; RMP or RPB5-mediating protein, interacts with RPB5 and counteracts transactivation by $\mathrm{HBx}[6,7]$; RPB5 binds general transcription factor IIB and together with $\mathrm{HBx}$ forms a ternary complex[8, 9]. Evidence revealed that $\mathrm{RPB} 5$ is directly involved in activated transcription[10]. TFIIF and RAP30 have been shown to associate with pol II and recruit pol II to the promoter in the transcription initiation[11-13]. Recent report demonstrates that RPB5 is the responsible subunit of pol II to associate with subunit RAP30 of TFIIF complex[14].

TFIIF is a general transcription factor which functions in both transcription initiation and elongation[11, 15]. Eukaryotic TFIIF is a heteromeric tetramer of RAP30 and RAP74 which have been isolated as RNA polymerase II-associated proteins (RAP) by an affinity column containing immobilized pol II[16]. The structure and function of both RAP30 and RAP74 subunits of TFIIF have been well defined[15, 17-20]. The N-terminus of RAP30 is proposed to associate with RAP74 to form the TFIIF complex and is necessary for transcription initiation $[16,21,22]$. The middle part of RAP30 is a sigmahomologous region that associates with pol II through binding to RPB5 and is essential for transcription elongation[23]. The C-terminus of RAP30 contains a cryptic DNA-binding domain, which is suggested to be homologous to the DNA template-binding domain of prokaryotic sigma factor[13]. TFIIF has been shown to be necessary for most, if not all, preinitiation complex formation and gene transcription[24-27].

TFIIF communicates with a number of factors to regulate gene transcription. It was reported that TFIIF directly bind to basal factors of TFIID, TFIIE and TFIIB[21]. Transcriptional activators, such as serum response factor (SRF), have been demonstrated to bind RAP74 in the middle of the molecule. On promoters such as adenovirus major late promoter, RAP74 subunit helps to wrap the DNA approximately one turn around the general transcription factors and RNA polymerase II. The RAP30 component of TFIIF can enhance the assembly of pol II into the initiation complex and RAP74 binding to the initiation complex will allow pol II to make promoter contact[28, 29].
RMP was identified as a protein with 508 amino acids. Various mammalian tissues have been found to ubiquitously express RMP. RMP interacts with RPB5 and counteracts transactivation by HBx. Immunoprecipitation demonstrated that RMP associate with assembled pol II complex and probably is an integral component of pol II holoenzyme. RMP has also been found to suppress activated transcription by VP16[6].

As both RMP and TFIIF tightly associate with RPB5, RMP and TFIIF must be spatially close and an interaction between them is therefore possible during the preinitiation complex formation and gene transcription. Effort was made to elucidate the possible interaction between TFIIF and RMP with its effect on activated transcription.

\section{MATERIALS AND METHODS}

\section{Plasmids constructions}

The plasmids pNKFLAG and pNKGST, derived from pSG5UTPL, are FLAG-tagged and GST-tagged mammalian expression vectors, respectively, as reported[30]. The plasmids pGENK1 and pGENKS are bacterial expression vectors for GSTfused proteins as reported previously[9].

The construction of full-length and truncated RMP expression plasmids have been described[6]. The internal deletion mutant RMP/Id5 was generated by splicing PCR using a primer set of 5'-GCGAGCTCCATG AGG CTA GGA AAT GTA-3' and 5'ATT TTC TTG CTC AAC AGT ATG TGA AAA ATA TAT-3' together with an other primer set of 5'-ACT GTT GAG CAA GAA AAT CAA AAG AAA CTT TTG-3' and 5'-GCGGATCCGTC TTT CTG TTG CAA-3'. The PCR product containing an artificial SacI site and a BamHI site was digested and inserted into pGENK1 and pNKFLAG. The plasmids pGST-RAP30 and His-ET-TAP74 were a gift from R.G Roeder. The other RAP30 and RAP74 or their translation expression plasmids were reported[14].

\section{Preparation of recombinant proteins}

GST-fused proteins were expressed in Escherichia coli by induction with $0.4 \mathrm{mM}$ isopropyl-D-thio-galactopyranoside at 30 ${ }^{\circ} \mathrm{C}$ for $3 \mathrm{~h}$. The cells were harvested and sonicated in PBST buffer (phosphate-buffered saline containing 1\% Triton X-100) $(4,5)$. After centrifugation, the extracts (supernatants) were collected and stored at $-80^{\circ} \mathrm{C}$. For purification, the extracts were incubated with glutathione-Sepharose 4B (Amersham Pharmacia Biotech) at room temperature for $1 \mathrm{~h}$. The beads were precipitated, washed four times with an excess amount of PBST buffer, and then eluted with $10 \mathrm{mM}$ reduced glutathione in $50 \mathrm{mM}$ Tris-HCI ( $\mathrm{pH} 8$. 0 ). The eluted proteins were divided into aliquots and stored at $80^{\circ} \mathrm{C}$.

FLAG-tagged proteins were expressed in BL21 by induction with $0.4 \mathrm{mM}$ isopropyl-D-thiogalactopyranoside at $30^{\circ} \mathrm{C}$ for $3-6 \mathrm{~h}$. 
The cells were harvested and sonicated in $50 \mathrm{mM}$ Tris-HCI, $\mathrm{pH} 8$. $0,150 \mathrm{mM} \mathrm{NaCI}$, and $0.1 \%$ Triton X-100. After centrifugation, the supernatant was collected and stored at $-80^{\circ} \mathrm{C}$. FLAG-tagged proteins were purified by incubating the sonication supernatant with anti-FLAG M2 resin (Kodak Scientific Imaging Systems) followed by several washes. The bound proteins were eluted with buffer containing FLAG peptide $(0.2 \mathrm{mg} / \mathrm{ml}$ FLAG peptide, $50 \mathrm{mM}$ Tris-HCI, $\mathrm{pH}$ 8.0, $150 \mathrm{mM} \mathrm{NaCl}$ ).

\section{In vitro GST resin pull-down assays}

GST resin pull-down assay was carried out as reported[6, 8, 9, 14]. Approximately $1 \mu \mathrm{g}$ of GST or GST-fused protein immobilized on $20 \mu \mathrm{l}$ of glutathione-Sepharose $4 \mathrm{~B}$ preblocked in $0.5 \%$ nonfat milk and $0.05 \%$ bovine serum albumin was incubated with $0.1 \mu \mathrm{g}$ of FLAG-tagged proteins in $0.5 \mathrm{ml}$ of modified GBT buffer for 1$2 \mathrm{~h}$ on a rotator at $4 \mathrm{oC}$. After being washed four times with modified GBT buffer, the bound proteins were eluted, fractionated by $12.5 \%$ SDS-PAGE, and subjected to Western blot analysis using anti-FLAG monoclonal antibody (M2).

\section{Immunoprecipitation and western blot analysis}

Transient transfection of COS1 cells was carried out as reported previously[1, 7, 8]. The cells were harvested, washed, and sonicated in LAC buffer (10\% glycerol, $20 \mathrm{mM}$ HEPES, $\mathrm{pH}$ 7.9, 50 $\mathrm{mM} \mathrm{KCI}, 0.4 \mathrm{M} \mathrm{NaCI}, 1 \mathrm{~m} M \mathrm{MgCl}, 0.1 \mathrm{~m} M$ dithiothreitol, $0.1 \mathrm{~m} M$ EDTA, $9 \mathrm{~m} M$ CHAPS, $0.5 \mathrm{~m} M$ phenylmethylsulfonyl fluoride, 10 $\mu \mathrm{g} / \mathrm{ml}$ aprotinin and leupeptin) and then centrifuged. The total cell lysates were stored at $-80^{\circ} \mathrm{C}$. Approximately $1.5 \mathrm{mg}$ of protein of total cell lysates was diluted with 4 times the volume of TBST buffer (50 mM Tris-HCI, pH 7.5, $50 \mathrm{mM} \mathrm{NaCl}, 0.05 \%$ Tween 20) and incubated with $50 \mu \mathrm{l}$ of packed Sepharose 4B for $30 \mathrm{~min}$. The supernatant was then obtained by centrifugation. Supernatants with FLAG-tagged proteins were immunoprecipitated with $20 \mu \mathrm{l}$ of $50 \%$ anti-FLAG M2 resin, rotated for $2 \mathrm{~h}$ at $4 \mathrm{oC}$, and washed four times with washing buffer TBS ( $50 \mathrm{~m} M$ Tris-HCI, $\mathrm{pH}$ 7.5, $150 \mathrm{mM}$ $\mathrm{NaCl})$. The bound proteins were eluted, fractionated by $12.5 \%$ SDS-PAGE, transferred onto nitrocellulose membranes, and subjected to Western blot analysis with the antibody. The proteins were visualized by enhanced chemiluminescence (ECL), according to the manufacturer's instructions (Amersham Pharmacia Biotech).

\section{Far-Western detection}

The far-Western blotting was performed as previously described[11]. The target protein (200 ng each) were fractionated on SDS-PAGE gels and then electrotransfered onto nitrocellulose membranes, which were denatured, renatured, and blocked with $5 \%$ skim milk in modified GBT buffer before subjected to farWestern blot[7]. The binding reaction proceeded in modified GBT buffer containing ${ }^{32} \mathrm{P}$-labeled probe $(40-100 \mathrm{ng} / \mathrm{ml}$ of protein with $2 \times 10 \mathrm{cpm} / \mu$ g of protein), $1 \% \mathrm{BSA}, 2 \mathrm{mM}$ of unlabeled ATP, and the sonicated supernatant of E. Coli JM109 transformed by pGENK1, which contained a final GST protein concentration of $1 \mathrm{mg} / \mathrm{ml}$. The membranes were washed five times with modified GBT buffer and exposed to X-ray films (XAR Omat, Kodak) or imaging plates
(Fuji).

\section{Transfection and luciferase assay}

Cell culture and transient transfection were carried out as reported previously $[9,30]$. Luciferase assay was conducted using dual-luciferase reporter system according to the manufacturer's instructions (Promega). Briefly 100ng of experiment reporter $\mathrm{pFRu}$-luc and $10 \mathrm{ng}$ of control reporter $\mathrm{pRu}$-luc were co-transfected with 5-20 ng of Gal-VP16 and 0-2 $\mu \mathrm{g}$ of RMP expression plasmids. Luciferase values were corrected for differences in apparent transfection efficiency by expression as a ratio with Renilla luciferase signals in the corresponding samples to normalize the transfection efficiency.

\section{RESULTS}

\section{RMP associates with TFIIF in vitro}

As RPB5 associates with both RMP and TFIIF, we examined the possible interaction between the full-sized RMP and each subunits of TFIIF in vitro. GST-fused RMP was expressed in E. Coli, purified by glutathione Sepharose 4B and fractionated by SDSPAGE (Fig 1B, lane 1). Flag-tagged RAP74 and RAP30 were expressed in E.coli and purified as described in Material and Methods. Pull-down assay was carried out with GST-RMP and FLAGRAP30 or FLAG-RAP74. The result demonstrated that both RAP74 and RAP30 in Flag-fused form associated with full-sized RMP in GST-fused form. Although as a negative control, GST bound neither FLAG-RAP30 nor FLAG-RAP74, the FLAG-RPB5, as a positive control, strongly bound GST-RMP (Fig 2, lane 1 and lane 10), confirming the previous result by Dorjisuren et al.

\section{Both subunits of TFIIF interact with the same region of $R M P$}

As the full RMP contains several domains which have been elucidated for the bindings with RPB5 and TFIIB $[6,7]$, we tried to delineate binding regions of RMP for the association with TFIIF subunits. A series of constructions were made to express various trancation mutants of RMP in GSTfused form. The bacterially expressed proteins of RMP mutants were purified and fractionated by SDS-PAGE as shown in Fig 1.

To map the binding region of RMP, pull-down assay was carried out with various trancation mutants of RMP in GST-fused form and RAP74 or RAP30 in Flag-fused form. The results were shown in Fig $2 \mathrm{~A}$ and 2B. Interestingly RAP74 and RAP30 showed a similar binding pattern with RMP. In another word, D5 and D10 of RMP bound both FlagRAP74 and Flag-RAP30 while other trancation mutants of RMP in GST-fused form or GST alone bound neither Flag-RAP74 nor Flag-RAP30. D5 is 
the minimal binding region in RMP for the interaction with both RAP74 and RAP30. To confirm the result we also set a positive control of binding between RPB5 and RMP which has been well defined [6]. There was a very strong binding between Flag-

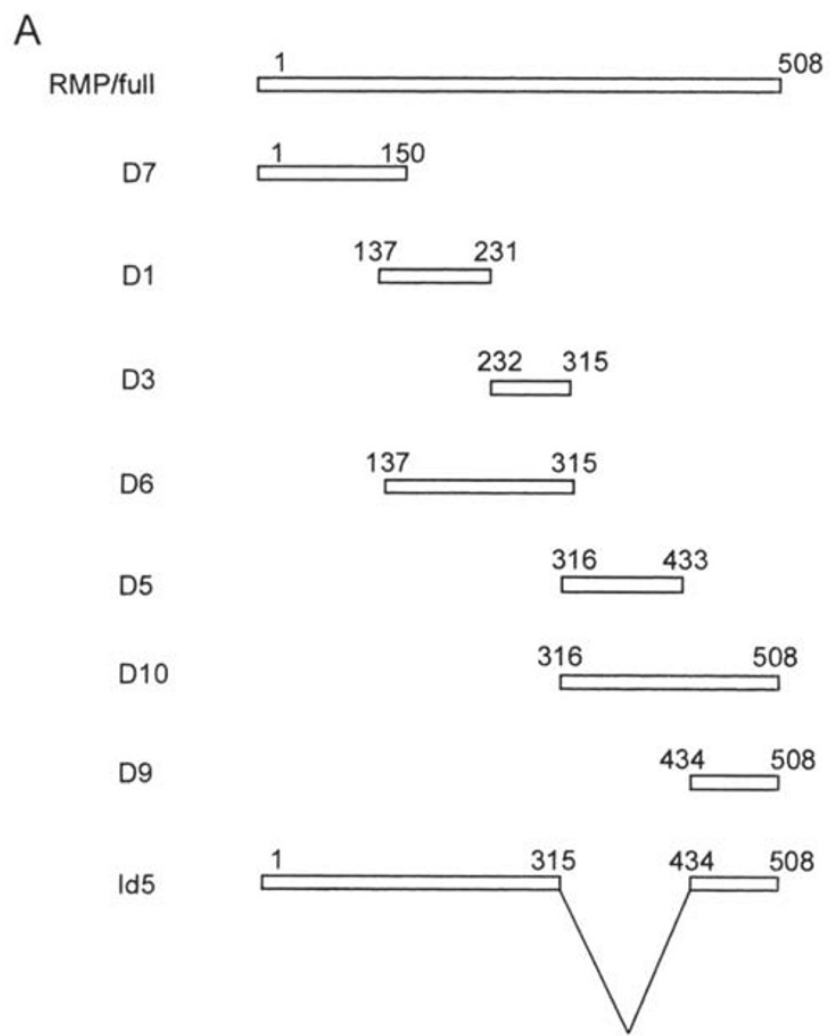

B

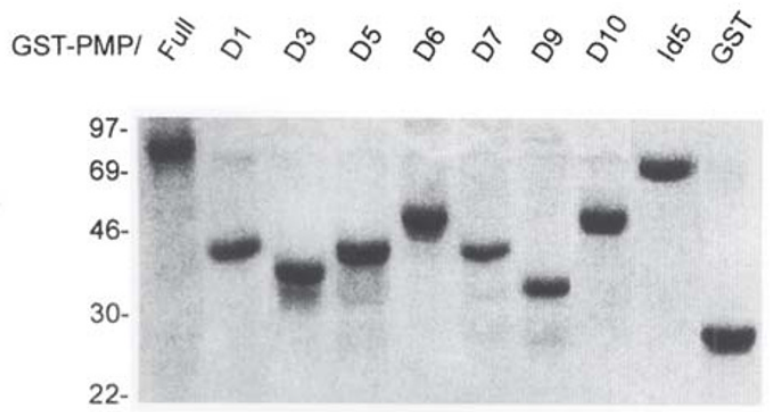

Fig 1. Delineation and purification of RMP proteins. A. Schematic map of various RMP deletion constructs. The expression plasmids were constructed as described in "Materials and Methods". B. Various GST-fused RMP truncation proteins were expressed in E. Coli, purified, fractionated by 12 . 5\% SDS-PAGE as described under "Materials and Methods" and visualized by Coomassie Brilliant Blue staining. The
RPB5 and full-sized RMP in GST-fused form (Fig $2 \mathrm{C}$, lane 1). Both D6 and D1 of RMP trancation mutants bound Flag-RPB5 although D1 was the minimal region for RPB5-binding, which is consistent with the previous report[6]. The binding pattern of RMP with RPB5 is different from that of RMP with TFIIF subunits in which RAP30 and RAP74 bound to the same region of RMP.

D5 region is required and sufficient for the association between RMP and TFIIF

To further confirm that D5 region of RMP bound both subunits of TFIIF, we also did Far-Western blotting. In addition to other trancation mutants of RMP used in Fig 2, we constructed a mutant with an internal deletion of D5 region (from residual 315 to 413) in RMP. Bacterially expressed RMP or its mutants in GST-fused form was purified, fractionated and transferred to nitrocellulose membrane. After being denatured and renatured, the membrane was subjected to Far-western detection by GSTRAP30 or GST-RAP74 probe. Although full RMP in GST-fused form bound both GST-RAP30 and GST-RAP74 probes, the internal deletion of D5 region abolished its ability to bind GST-RAP30 and GST-RAP74. The D5 region alone was sufficient to associate with GST-RAP30 and GST-RAP74. The other trancation mutants of GST-RMP together with GST alone bound neither RAP30 nor RAP74 in the Far-Western detection, consistent with the result of pull-down assay (Fig 3). The results suggest that D5 region is necessary and sufficient for the association between RMP and RAP74 or RAP30.

\section{RMP associates with TFIIF in vivo}

Although the result of pull-down assay and Farwestern detection were consistent, both are in vitro binding assay which may not reflect the physiological situation in vivo. Therefore we performed immunoprecipitation to observe the association between RMP and RAP74 or RAP30 in vivo. COS1 cells were cotransfected with mammalian expression plasmids of Flag-RMP or its trancation mutants and GST-RAP74 or GST-RAP30. The proteins were expressed approximately equally in the transfection as shown in Fig 4A. Immunoprecipitation was carried out with anti-Flag M2 antibody-bound resin and 


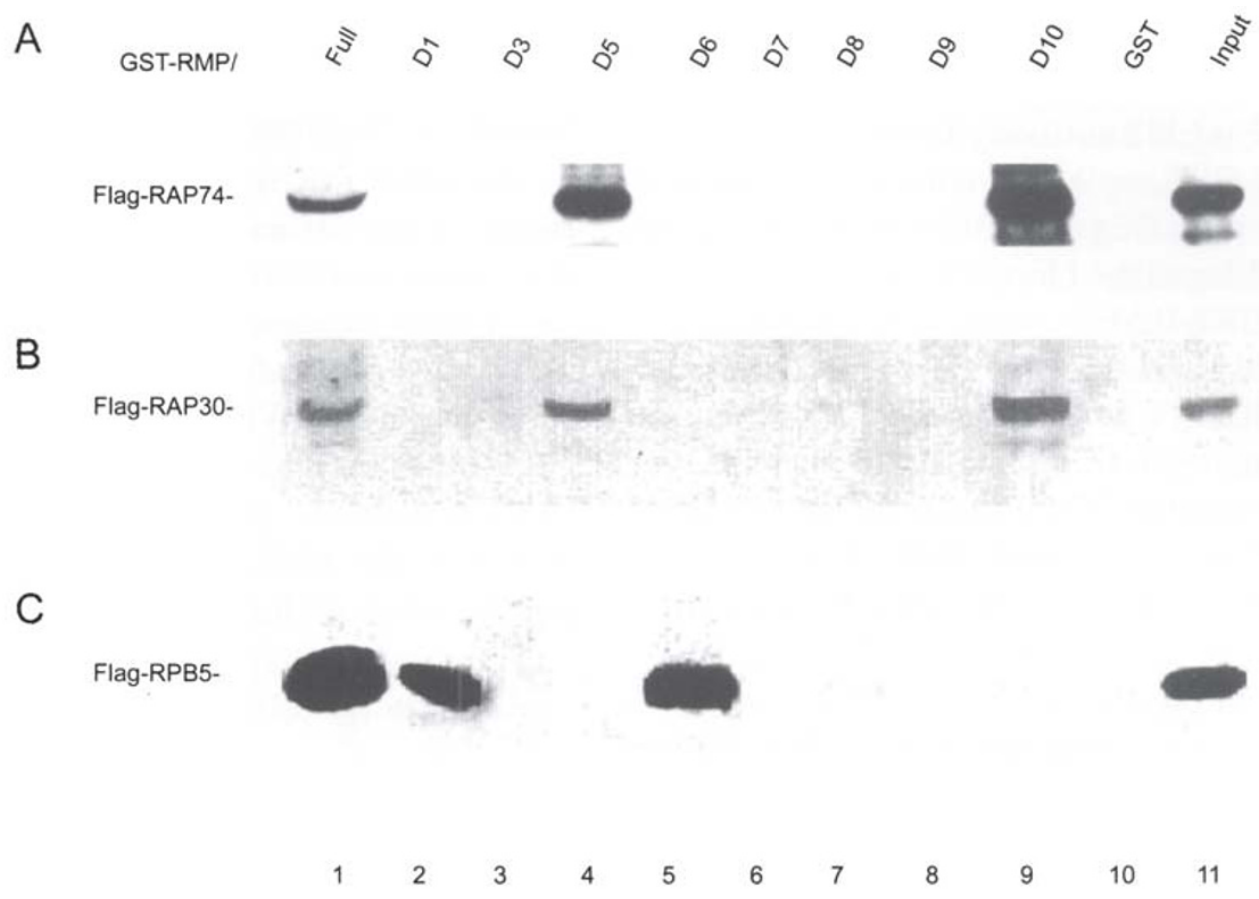

Fig 2. GST resin pull-down assay. Approximately $1 \mu \mathrm{g}$ of GST (lane 10) or GST-fused RMP truncation proteins purified from bacterial expression was immobilized on glutathione resin and incubated with $0.1 \mu \mathrm{g}$ of bacterially expressed FLAG-RAP74 (A), or FLAG-RAP30 (B), or FLAG-RPB5 (C) in GBT buffer. Pull-down assay and Western blot analysis were carried out with anti-FLAG M2 antibody as described under "Materials and Methods". Lane 11 shows 5\% of the input of FLAG-tagged proteins.

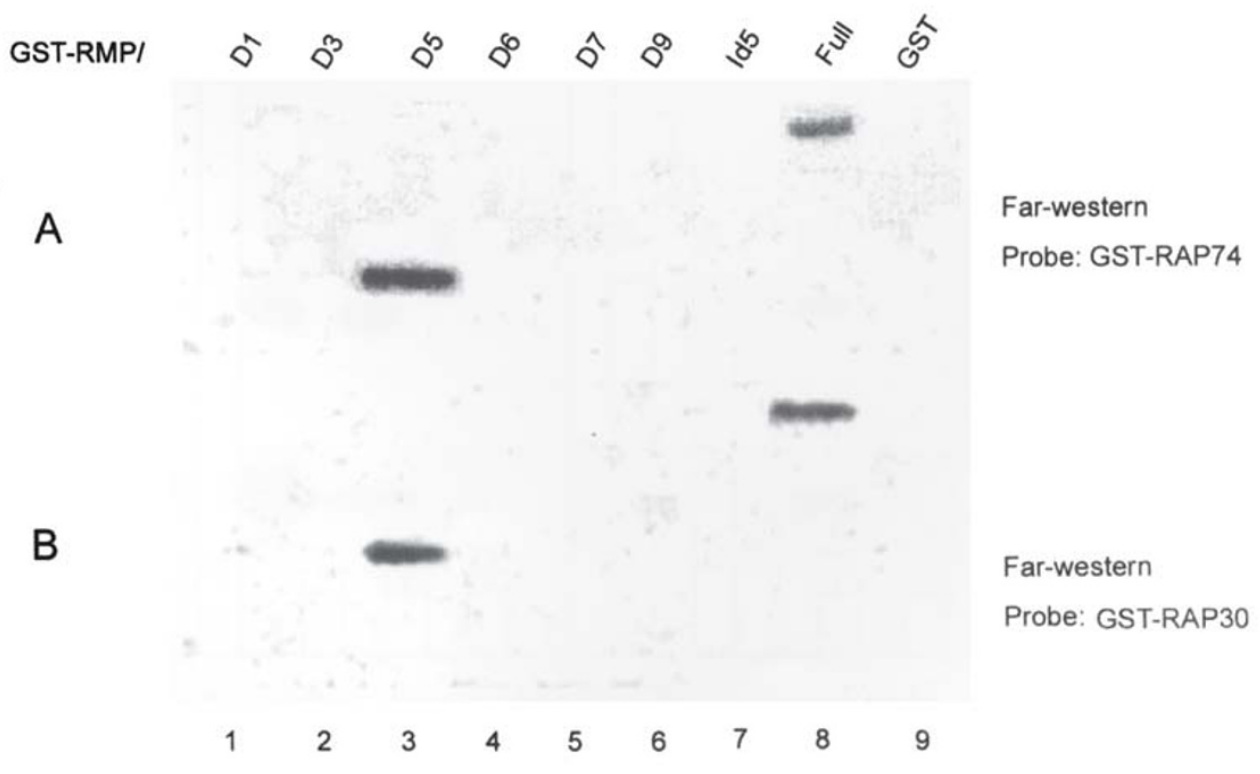

Fig 3. RMP binding with TFIIF detected by Far-western blotting. Equal amounts of GST (lane 9) or GST-fused RMP truncation proteins (200 ng each) was fractionated by $12.5 \%$ SDS-PAGE and electrotransferred onto nitrocellulose membranes. After denature, renature and being blocked by $5 \%$ skim milk in modified GBT buffer, the membranes were subjected to Far-western detection with ${ }^{32}$ P-labed probes of GST-RAP74 (A) or GST-RAP30 (B). 
detected by anti-GST antibody. The results matched with that of in vitro (Fig 4B). Full-sized RMP and its D5 mutant in Flag-tagged form recovered both GST-RAP74 and GST-RAP30 while other mutants including Id5 in which D5 region was deleted recovered neither GST-RAP74 nor GST-RAP30. And apparently the immunoprecipitated RAP74 and RAP30 were in approximately equal molar ration, suggesting that RMP associated with RAP74 and RAP30 which were in the form of TFIIF complex rather than individual subunits.
Interaction with TFIIF is required for the RMPmediated suppression of activated transcription

As RMP was reported to functions as a corepressor of the activated transcription, we wonder if the interaction between RMP and TFIIF is necessary for the suppression function of RMP. Therefore we examined the effects of RMP on activated transcription by Gal-VP16, a chimeric activator with Gal4 DNA binding domain fused to the VP16 activation domain. $\mathrm{pFRu}$-luc luciferase reporter was driven by five Gal4 binding sites. COS1 cells were

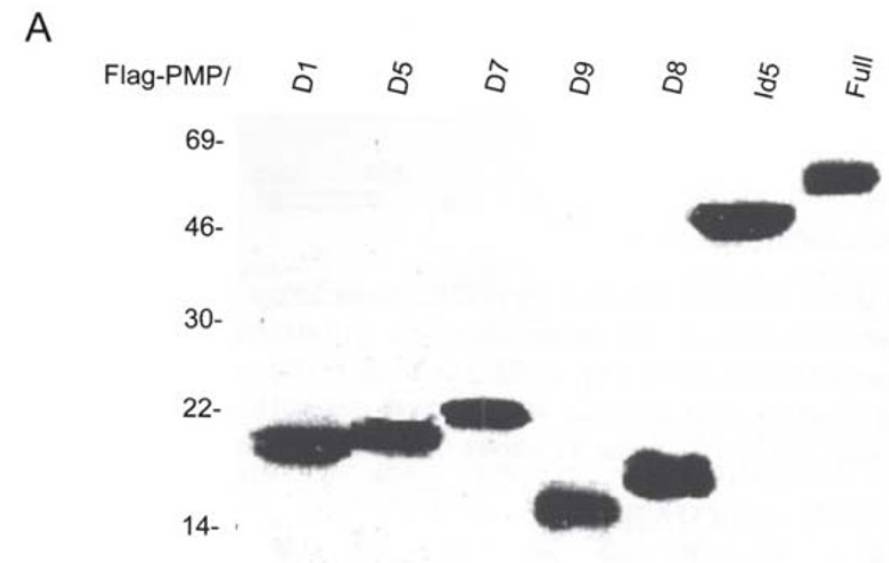

B

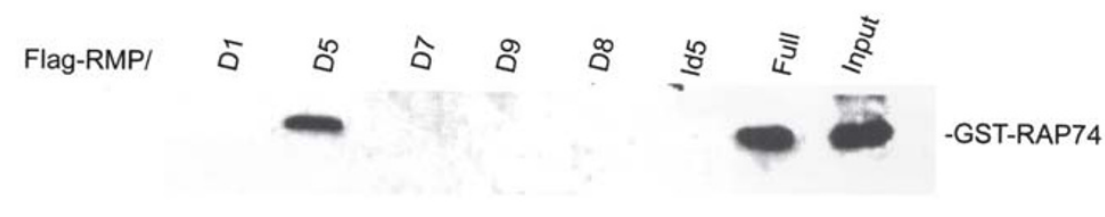

C

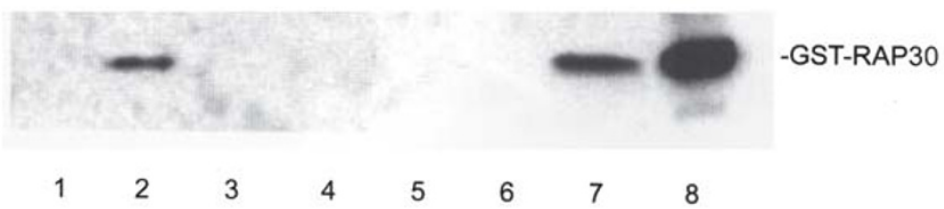

Fig 4. RMP associates with TFIIF in vivo. COS 1 cells were cotransfected with mammalian expression plasmids pNKFLAG-RMP and pNKGST-RAP74 or pNKGST-RAP30. Cell lysates were prepared as described under "Materials and Methods". Approximately $1.5 \mathrm{mg}$ proteins of total lysate was immunoprecipitated with $20 \mu \mathrm{l}$ of packed anti-FLAG M2 antibody-bound resin. After being washed, the bound proteins were eluted and then fractionated by $12.5 \%$ SDSPAGE and subjected to Western blot analysis with anti-FLAG M5 antibody (A) or anti-GST antibody (B and C). Lane 8 of panel B and C shows $5 \%$ of the input of total lysate used in lane 4. 
cotransfected with expression plasmids of reporter, Gal-VP16 and RMP or its mutants. The results of the luciferase assay demonstrated that the activated transcription by Gal-VP16 was suppressed by RMP, which is consistent with the results by CAT assay [6]. However RMP-Id5 (in which TFIIF-binding region was deleted) lost the suppression function for the activated transcription by Gal-VP16 (Fig 5). The results show that the association with TFIIF is necessary for the RMP suppression function of activated transcription.

\section{DISCUSSION}

Most RNA polymerase subunits are unique to their respective RNA polymerases, while some subunits are common among RNA polymerses I, II, and III. With the exception of RPB5, all of the common subunits in human RNA polymerases I, II, and III can substitute for their yeast homologues, although RPB5 is highly conserved among humans, Saccharomyces cerevisiae, and Schizosaccharomyces pombe, and is essential in yeast[2, 31, 32]. Yeast RPB5 has been reported to interact with RPB3 in vitro, and both RPB5 and RPB3 are present in two molar amounts in RNA polymerase II and seem to play an important role in subunit assembly, as does human RPB5.

Previously we identified that $\mathrm{HBx}$, the multifunctional viral regulator protein of hepatitis $B$ virus, directly target RPB5 of RNA polymerase II[4, 5]. And both RPB5 and HBx communicate with TFIIB. The trimeric interaction of these three factors may facilitate transcription and $\mathrm{HBx}$ acts as coactivator in activated transcription[8, 9]. Based on these

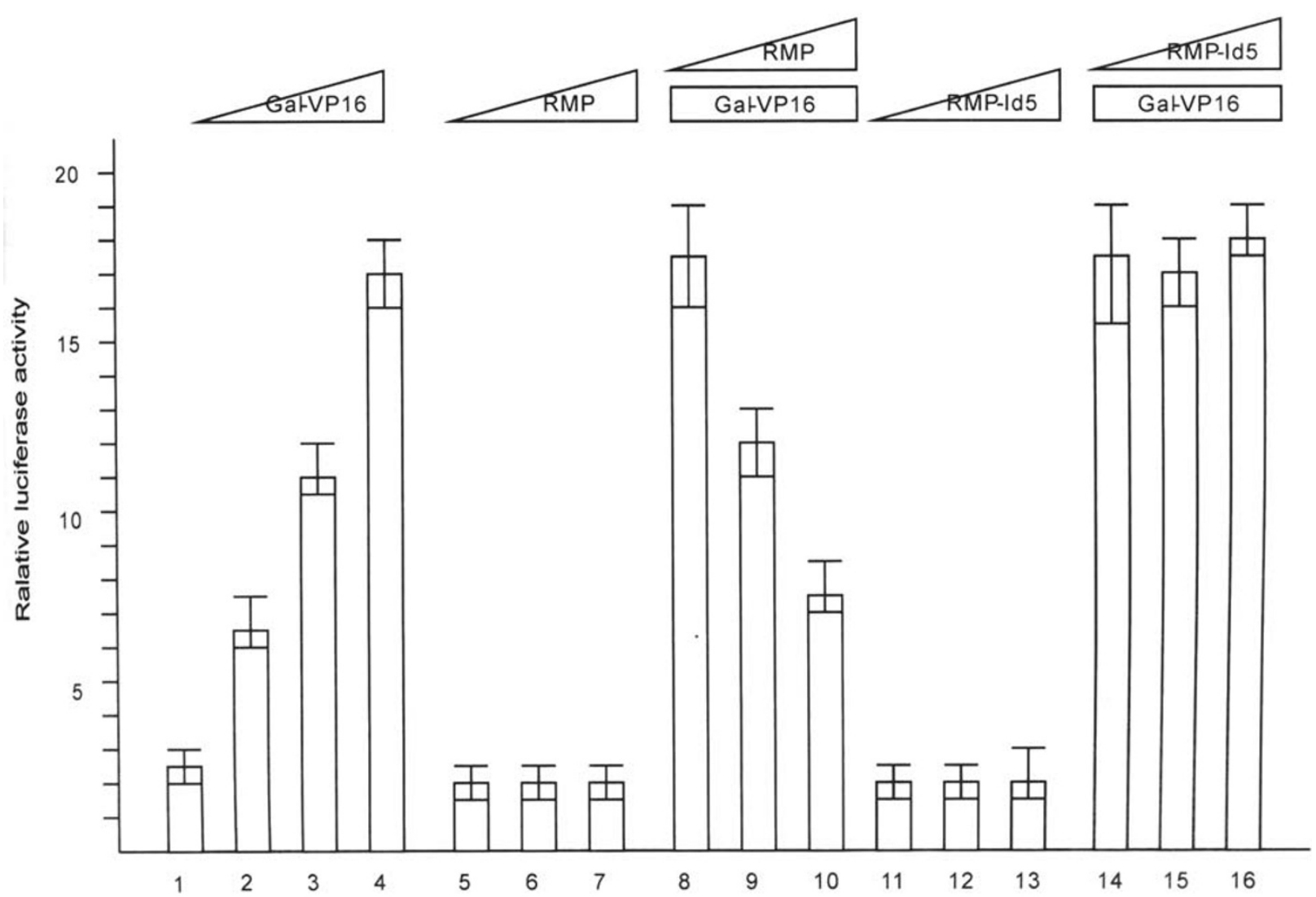

Fig 5. D5 region of RMP is required to suppress activated transcription. COS1 cells were cotransfected with pGalLuc and Gal-VP16 together with RMP or RMP-Id5 constructs. The transfected plasmid DNA was $0.1 \mu \mathrm{g}$ of pGalLuc together with the following: bars 1 to 4, 0, 5, 10, and $15 \mathrm{ng}$ of pGal-VP16, respectively; bar 5 to 7, 0, 1, and $2 \mu \mathrm{g}$ of pSG5UTPLRMP, respectively; bar 8 to 10, 0, 1, and $2 \mu$ g of pSG5UTPL-RMP, respectively, plus 15 ng of pGal-VP16; bar 11 to 13, 0,1 , and $2 \mu \mathrm{g}$ of pSG5UTPL-RMP-Id5, respectively; bar 14 to 16, 0, 1, and $2 \mu \mathrm{g}$ of pSG5UTPL-RMP-Id5, respectively, plus $15 \mathrm{ng}$ of pGal-VP16. The total amount of DNA added per transfection was adjusted to $10 \mu \mathrm{g}$ with the control vector, pSG5UTPL. Error bars shows standard deviations. 
results, we proposed that RPB5 is a communicating subunit of pol II that interacts with transcriptional regulators. In support of the notion, we identified a novel protein, RPB5-mediating protein (RMP), which counteracts the coactivator function of $\mathrm{HBx}$ by competitively binding RPB5[6, 7]. The crystal structure of pol II revealed that RPB5 is composed of the exposed domain of $\mathrm{N}$-terminal and the embedded C-terminal domain. The exposed domain is close to the DNA template and responsible for transcription regulation by interaction with transcription factors, such as TFIIB and RMP[33, 34].

RMP was originally identified by its association with RPB5. RMP has been shown to counteract HBx transactivation and the increased amount of $\mathrm{HBx}$ reduced the suppression effect of RMP. In the in vitro and in vivo binding assay RPB5 and HBx competed with each other to associate with RMP. The activated transcription by Gal-VP16 was suppressed by RMP in mammalian cells. Based upon these observations RMP has been suggested to function as a corepressor of transcription regulator[6].

In this report D5 region of C-terminal RMP has been shown to interact with both subunits of TFIIF, which has been confirmed by pull-down assay, Farwestern blot and immunoprecipitation. It is possible that RAP30 and RAP74 contact the different surfaces of D5 region of RMP. Or the RMP-interacting surfaces of RAP30 and RAP74 are very closely positioned within the D5 region so that further fine delineation could possibly separate the RAP30 and RAP74-binding domains. The fact that D5 region associates with both subunits of TFIIF may suggest a function of RMP in the regulation of formation of TFIIF complex by associating with both subunits of RAP30 and RAP74.

It was reported that RMP also interact with TFIIB by two independent domains of the N-terminal region D8 and the C-terminal domain D10 of RMP which overlaps with TFIIF binding region (7 and unpublished data). D5 region of RMP seems to be the minimal region of C-terminal RMP for TFIIBbinding as the D9 region from residual 434 to 508 was negative of TFIIB-binding and D10 region is positive for the binding. It could be deduced that the region of RMP from residual 316 to 434 is responsible for the association between C-terminal RMP and TFIIB. This is an interesting phenom- enon as this D5 region of RMP from residual 316 to 434 is exactly the common domain to interact with TFIIB and TFIIF. The relationship and mechanism among RMP, TFIIB and TFIIF remain to be addressed. One possibility is that the two general transcription factors of TFIIF and TFIIB compete with each other to bind RMP and positively or negatively regulate the suppression function of RMP. RMP may also serve as a scaffold for TFIIF and TFIIB to communicate with RPB5 of pol II.

In addition to associating with RPB5, TFIIB and TFIIF, RMP also bind with pol II complex[6], suggesting that RMP may be a necessary component of pol II holoenzyme complex. So it is possible that RMP regulates transcription from pol II holoenzyme complex by interacting with many different factors except RPB5 and TFIIB. In this report we demonstrated that both subunits of TFIIF interacted with RMP, which may be an additional way of transcription regulation by RMP. Alternatively TFIIF may be a necessary component for the suppression function of RMP through interaction with RPB5 and/or TFIIB. Actually as RMP, TFIIF, TFIIB and RPB5 bind with each other, they may form a complex and cooperatively regulate the transcription.

Accumulated evidences appear that transcription is regulated by factors through targeting TFIIF. A number of activators enhance the transcription by its association with RAP74 or RAP30[26, 27, 35]. Recently the interaction between heat shock factor4 , a transcriptional repressor, with the basal transcription factor TFIIF was firstly identified[48].

We report here that RMP interacts with TFIIF and suppresses the activated transcription by GalVP16. Disassociation of RMP and TFIIF by the internal deletion of TFIIF-binding region in RMP abolished suppression function of RMP. A similar phenomenon was observed in the interaction between the RMP and RPB5. Disruption of the association between RMP and RPB5 results in loss of suppression function of RMP, which suggests that contacting with both RPB5 and TFIIF are necessary for the suppression function of RMP. It has been shown that pol II is associated with both RMP and TFIIF[6, 14]. Destruction of the conformation of the holoenzyme complex by disassociation of RMP with TFIIF or RPB5 leads to the loss of suppression function of RMP. The interaction between TFIIF and 
RMP could be either an independent passway for RMP to regulate the transcription or a necessary part of network with TFIIB, RPB5 and pol II for the corepressor function of RMP, which remain to be addressed.

\section{ACKNOWLEDGEMENTS}

We thank Dr. RG Roeder for the plasmids of HispET-RAP74 and pGST-RAP30. We are grateful to Dr. Y. Hirose for the plasmids of $\mathrm{pFRu}$-luc and $\mathrm{pRu}-$ luc; and to F. Momoshima, M. Yasukawa, and K. Kuwabara for technical assistance. We also thank Dr. E Sun for encouraging discussion.

This work was partly supported by National Natural Science Foundation of China (Grant 30140007).

\section{REFERENCES}

1. Schlegel BP, Green VJ, Ladias JA, Parvin JD. BRCA1 interaction with RNA polymerase II reveals a role for hRPB2 and hRPB10alpha in activated transcription. Proc Natl Acad Sci USA 2000; 97:3148-53. Tan

2. Q, Linask KL, Ebright RH, Woychik NA. Activation mutants in yeast RNA polymerase II subunit RPB3 provide evidence for a structurally conserved surface required for activation in eukaryotes and bacteria. Genes Dev 2000; 14:339-48.

3. Koleske AJ, Young RA. An RNA polymerase II holoenzyme responsive to activators. Nature 1994; 368:466-9.

4. Murakami S. Hepatitis B virus X protein: structure, function and biology. Intervirology 1999; 42:81-99.

5. Cheong JH, Yi M, Lin Y, Murakami S. Human RPB5, a subunit shared by eukaryotic nuclear RNA polymerases, binds human hepatitis B virus $\mathrm{X}$ protein and may play a role in X transactivation. Embo J 1995; 14:143-50.

6. Dorjsuren D, Lin Y, Wei W, Yamashita T, Nomura T, Hayashi N, Murakami S. RMP, a novel RNA polymerase II subunit 5-interacting protein, counteracts transactivation by hepatitis B virus $\mathrm{X}$ protein. Mol Cell Biol 1998; 18:7546-55.

7. Yang Y, Zheng L, Chen Y. [Study of HBV X protein and RMP, an RPB5 mediate protein competitively interacting with general transcription factor TF2B]. Zhonghua Gan Zang Bing Za Zhi 2000; 8:15-7.

8. Lin Y, Tang H, Nomura T, Dorjsuren D, Hayashi N, Wei W, Ohta T, Roeder R, Murakami, S. The hepatitis B virus $\mathrm{X}$ protein is a co-activator of activated transcription that modulates the transcription machinery and distal binding activators. J Biol Chem 1998; 273:27097-103.

9. Lin Y, Nomura T, Cheong J, Dorjsuren D, Iida K, Murakami S. Hepatitis B virus X protein is a transcriptional modulator that communicates with transcription factor IIB and the RNA polymerase II subunit 5. J Biol Chem 1997; 272:7132-9.
10. Miyao T, Woychik NA. RNA polymerase subunit RPB5 plays a role in transcriptional activation. Proc Natl Acad Sci USA 1998; 95:15281-6.

11. Roeder RG. The role of general initiation factors in transcription by RNA polymerase II. Trends Biochem Sci 1996; 21:327-35.

12. Garrett KP, Serizawa H, Hanley JP, Bradsher JN, Tsuboi A, Arai N, Yokota T, Arai K, Conaway RC, Conaway JW. The carboxyl terminus of RAP30 is similar in sequence to region 4 of bacterial sigma factors and is required for function. J Biol Chem 1992; 267:23942-9.

13. Sopta M, Burton ZF, Greenblatt J. Structure and associated DNA-helicase activity of a general transcription initiation factor that binds to RNA polymerase II. Nature 1989; 341:410-4.

14. Wei W, Dorjsuren D, Lin Y, Qin W, Nomura T, Hayashi N, Murakami S. Direct interaction between the subunit RAP30 of transcription factor IIF (TFIIF) and RNA polymerase subunit 5, which contributes to the association between TFIIF and RNA polymerase II. J Biol Chem 2001; 276:12266-73.

15. Nikolov DB, Burley SK. RNA polymerase II transcription initiation: a structural view. Proc Natl Acad Sci USA 1997; 94:15-22.

16. Sopta M, Carthew RW, Greenblatt, J. Isolation of three proteins that bind to mammalian RNA polymerase II. J Biol Chem 1985; 260:10353-60.

17. Conaway JW, Conaway RC. A multisubunit transcription factor essential for accurate initiation by RNA polymerase II. J Biol Chem 1989; 264:2357-62.

18. Flores O, Ha I, Reinberg D. Factors involved in specific transcription by mammalian RNA polymerase II. Purification and subunit composition of transcription factor IIF. J Biol Chem 1990; 265:5629-34.

19. Kim J B, Yamaguchi Y, Wada T, Handa H, Sharp PA. TatSF1 protein associates with RAP30 and human SPT5 proteins. Mol Cell Biol 1999; 19: 5960-8.

20. Lipinski KS, Esche H, Brockmann D. Amino acids 1-29 of the adenovirus serotypes 12 and 2 E1A proteins interact with rap30 (TF(II)F) and TBP in vitro. Virus Res 1998; 54:99-106.

21. McEwan IJ, Gustafsson J. Interaction of the human androgen receptor transactivation function with the general transcription factor TFIIF. Proc Natl Acad Sci USA 1997; 94:8485-90.

22. Tan S, Aso T, Conaway RC, Conaway JW. Roles for both the RAP30 and RAP74 subunits of transcription factor IIF in transcription initiation and elongation by RNA polymerase II. J Biol Chem 1994; 269:25684-91.

23. McCracken S, Greenblatt J. Related RNA polymerasebinding regions in human RAP30/74 and Escherichia coli sigma 70. Science 1991; 253:900-2.

24. Tan S, Conaway RC, Conaway JW. Dissection of transcription factor TFIIF functional domains required for initiation and elongation. Proc Natl Acad Sci USA 1995; 92 : 6042-6.

25. Flores O, Lu H, Killeen M, Greenblatt J, Burton ZF, Reinberg D. The small subunit of transcription factor IIF 
recruits RNA polymerase II into the preinitiation complex. Proc Natl Acad Sci USA 1991; 88:9999-10003.

26. Killeen M, Coulombe B, Greenblatt J. Recombinant TBP, transcription factor IIB, and RAP30 are sufficient for promoter recognition by mammalian RNA polymerase II. J Biol Chem 1992; 267:9463-6.

27. Killeen MT, Greenblatt JF. The general transcription factor RAP30 binds to RNA polymerase II and prevents it from binding nonspecifically to DNA. Mol Cell Biol 1992; 12:30-7.

28. Joliot V, Demma M, Prywes R. Interaction with RAP74 subunit of TFIIF is required for transcriptional activation by serum response factor. Nature 1995; 373:632-5.

29. Zhu H, Joliot V, Prywes R. Role of transcription factor TFIIF in serum response factor-activated transcription. J Biol Chem 1994; 269:3489-97.

30. Murakami S, Cheong J, Ohno S, Matsushima K, Kaneko $\mathrm{S}$. Transactivation of human hepatitis B virus $\mathrm{X}$ protein, $\mathrm{HBx}$, operates through a mechanism distinct from protein kinase $\mathrm{C}$ and okadaic acid activation pathways. Virology 1994; 199:243-6.
31. McKune K, Woychik NA. Functional substitution of an essential yeast RNA polymerase subunit by a highly conserved mammalian counterpart. Mol Cell Biol 1994; 14: 4155-9.

32. McKune K, Moore PA, Hull MW, Woychik NA. Six human RNA polymerase subunits functionally substitute for their yeast counterparts. Mol Cell Biol 1995; 15:6895900.

33. Todone F, Weinzierl RO, Brick P, Onesti S. Crystal structure of RPB5, a universal eukaryotic RNA polymerase subunit and transcription factor interaction target. Proc Natl Acad Sci USA 2000; 97:6306-10.

34. Cramer P, Bushnell DA, Fu J, Gnatt AL, Maier-Davis B, Thompson NE, Burgess RR, Edwards AM, David PR, Kornberg RD. Architecture of RNA polymerase II and implications for the transcription mechanism. Science 2000; 288:640-9.

35. Maldonado E, Drapkin R, Reinberg D. Purification of human RNA polymerase II and general transcription factors. Methods Enzymol 1996; 274:72-100 